\title{
La polinización en los sistemas de producción agrícola: revisión sistemática de la literatura
}

\author{
Pollination in agricultural systems: a systematic literature review \\ Marta García García1*, Leonardo Alberto Ríos Osorio², Javier Álvarez del Castillo ${ }^{3}$
}

\begin{abstract}
RESUMEN
La polinización es un proceso esencial para el correcto funcionamiento de los ecosistemas y la producción de alimentos, que se ha visto enormemente afectado por diversas actividades antrópicas, siendo cada vez mayor el número de investigaciones encaminadas a entender cómo funciona este proceso. El objetivo de este trabajo es describir las funciones clave que realizan los polinizadores y sus efectos, a través de una revisión sistemática de la literatura científica publicada a lo largo de los últimos diez años. La metodología utilizada consistió en la búsqueda de publicaciones originales en cinco bases de datos, su filtrado por criterios de inclusión y exclusión, y la posterior añadidura de literatura gris. Los resultados muestran una tendencia al establecimiento de objetivos, metodologías de investigación y zonas de estudios similares, así como al estudio predominante de grupos de polinizadores de tipo abejas y abejorros, y al análisis de la polinización desde el punto de vista de la complementariedad de nicho, los patrones de actividad diaria y las asociaciones polinizador-planta, usando medidas como la abundancia de forrajeo, la influencia interespecífica, la deposición de polen o la producción final de frutos y semillas. También se observa una preocupación cada vez mayor por los efectos de las distintas técnicas de manejo agrícola sobre el proceso polinizador y por la necesidad de implementar medidas de conservación adecuadas, concluyéndose que, a pesar de todo ello, sigue siendo necesario continuar avanzando en la investigación dentro de este campo para lograr alcanzar un entendimiento más profundo de la situación.
\end{abstract}

Palabras clave: polinizadores, efecto, función, ecosistemas agrícolas.

\begin{abstract}
Pollination is an essential process for the correct performance of ecosystems and food production, and has been extremely affected by human activities; understanding this process has been the subject of an increasing number of studies. The purpose of this work is to describe the key functions of pollinators and their effects through a systematic review of the scientific literature of the last ten years. Methodology used consisted of a comprehensive search for original papers in five different databases, filtering these results by inclusion and exclusion criteria, and then adding grey literature. The results of the revision suggest a tendency towards similar investigation subjects, similar methodologies, and similar study locations, as well as a predominant study of the bee and bumblebee-type of pollinators. They also show a recurrent analysis of the pollination process: studying it from the point of view of niche complementarity, circadian rhythm activities and pollinator-plant relationships, and using measures such as foraging abundance, interespecific influence, pollen deposition or fruit and seed set. Furthermore, we have observed an increased concern regarding the effects of different agricultural techniques on the pollination process and regarding the need to implement adequate conservation measures. We conclude that it's still necessary to continue investigating within this research field to achieve a deeper understanding of the situation.
\end{abstract}

Key words: pollinators, effect, function, agricultural ecosystems.

1 Instituto de Sostenibilidad, Universidad Politécnica de Cataluña-Barcelona Tech. Calle Jordi Girona No 31, Ed. TG, planta S1. 08034, Barcelona, España.

2 Grupo de Investigación Salud y Sostenibilidad. Escuela de Microbiología, Universidad de Antioquia. Calle 67 \# 53-108, Bloque 5, Oficina 135. Ciudad Universitaria. Medellín (Antioquia), Colombia. Apartado aéreo 1226.

3 Departamento de Ingeniería Mecánica, Universidad Politécnica de Cataluña-Barcelona Tech. Calle Colom No 11,08222 Terrassa, Barcelona, España.

* Autor para correspondencia: martagg@gmx.es

Fecha de Recepción: 16 Marzo, 2016.

Fecha de Aceptación: 13 Mayo, 2016. 


\section{Introducción}

La polinización, entendida como la transferencia de polen desde la parte masculina de una flor hasta la parte femenina de la misma u otra flor, es un proceso esencial para el mantenimiento de la viabilidad y la diversidad genética de las plantas con flor, además de mejorar la calidad y cantidad de semillas y frutos, así como de las características de la descendencia (Chautá-Mellizo et al., 2012; Vergara y Badano, 2009; Vilhena et al., 2012). Puede ser realizada de forma abiótica, mediante el transporte del polen por el viento o el agua, o biótica, empleando para ello a animales como vectores en el transporte (Bonilla, 2012; Pantoja et al., 2014). Diversos grupos, tales como invertebrados, aves o mamíferos, pueden actuar como polinizadores, movidos por la necesidad de encontrar recursos para su alimentación, desarrollo o reproducción (Bonilla, 2012).

Alrededor del 78\% de las especies de plantas con flor en climas templados y del $94 \%$ en climas tropicales se benefician del proceso de la polinización mediada por animales, lo que equivale a más del $87 \%$ de todas las especies de angiospermas conocidas (Bonilla, 2012; Mayer et al., 2011). En cultivos tropicales, el $70 \%$ de las 1.330 especies cultivadas se ve favorecido por estos polinizadores, mientras que en cultivos europeos el $84 \%$ de las 264 especies cultivadas dependen del proceso de polinización animal (Chautá-Mellizo et al., 2012; Klein et al., 2007). A nivel global, el $87 \%$ de las especies cultivadas, que representan un $35 \%$ del suministro global de alimentos, se ven beneficiadas por este proceso (Hoehn et al., 2008; Klatt et al., 2014; Mallinger y Gratton, 2015), traduciéndose estos datos en un valor económico de la polinización que asciende a más de 153 billones de euros, o al 9,5\% del total de la producción agrícola (Ricou et al., 2014; Vilhena et al., 2012).

Por otra parte, se estima que, dentro del $90 \%$ de la polinización que ocurre en plantas con flor en todo el mundo, un $67 \%$ es llevado a cabo por insectos, constituyéndose como el grupo de polinizadores más importante, tanto para especies de plantas silvestres como cultivadas (Bonilla, 2012; Fründ et al., 2013). El papel de los insectos polinizadores, y fundamentalmente el de las abejas, ha sido ampliamente demostrado para todo tipo cultivos (Rader et al., 2013), siendo cada vez mayor el número de investigaciones que se preocupan por la pérdida de estos organismos y de los servicios que proveen, mientras que son pocos los estudios que tratan de evaluar o explicar el porqué de su importancia y la necesidad de conservar el servicio de la polinización (Kremen et al., 2007). Existen algunos trabajos que van desde valoraciones de la riqueza de géneros o especies de polinizadores en una zona (Colteaux et al., 2013; Russo et al., 2015; Wojcik et al., 2008), las relaciones que se establecen entre especies de polinizadores (Badano y Vergara, 2011) o entre polinizadores y plantas (Vilhena et al., 2012), hasta estimaciones acerca la influencia de la polinización sobre la calidad del polen y del néctar (Mensah y Kudom, 2011; Pérez-Balam et al., 2012), la diversidad floral (Ricou et al., 2014) o la actividad de los polinizadores (Rader et al., 2013), pasando por la evaluación final de la producción de semillas y frutos en presencia de estos organismos (Bos et al., 2007; Chautá-Mellizo et al., 2012; Greenleaf y Kremen, 2006; Hoehn et al., 2008; Mallinger y Gratton, 2015; Rogers et al, 2013; Vergara y Badano, 2009). Aún así, estos estudios son pocos y se encuentran dispersos.

Por ello, el objetivo de esta investigación es describir el papel clave que juegan los polinizadores en los ecosistemas, las funciones que realizan y sus efectos, a través de una revisión sistemática de la literatura científica publicada a lo largo de los últimos diez años, con la cual se pretende agrupar la información existente, así como aportar nuevos datos y hallazgos sobre el tema que, a su vez, permitan el desarrollo de investigaciones futuras.

\section{Material y Métodos}

\section{Estrategia de búsqueda}

Para llevar a cabo este trabajo, se efectuó una búsqueda sistemática de información en las bases de datos Web of Science, Scopus, ScienceDirect, JSTOR y PubMed, por sensibilidad utilizando descriptores DeCS, por exhaustividad utilizando descriptores no DeCS, y por especificidad mediante la combinación de términos a través de operadores booleanos.

En primer lugar, y tomando como punto de partida la pregunta de investigación “ ¿cuál es el efecto y la función de los polinizadores en los sistemas de producción agrícola?", se definió una ruta de búsqueda general [(pollination OR pollinator) AND (agroecology OR agroecosystems) AND (effect OR function)], y se acotó la búsqueda mediante unos 
límites de tiempo para abarcar la literatura publicada entre los años 2005 a 2015. Para cada base de datos, se ajustó tanto la ruta de búsqueda como el intervalo de tiempo. En Scopus y ScienceDirect se utilizó el límite de tiempo "2005 to present", mientras que en Web of Science, JSTOR y PubMed se emplearon los límites "2005-2015", "from 2005 to 2015" y "from 2005-01-01 to 2015-12-31", respectivamente. Las rutas de búsqueda específicas para cada base de datos son las siguientes: Web of Science $[\mathrm{TS}=(($ pollination OR pollinator) AND (agroecology OR agroecosystems) AND (effect OR function))]; Scopus [TITLE-ABS-KEY ((pollination OR pollinator) AND (agroecology OR agroecosystems) AND (effect OR function)) AND DOCTYPE (ar) AND PUBYEAR > 2004]; ScienceDirect [TITLEABS-KEY ((pollination OR pollinator) AND (agroecology OR agroecosystems) AND (effect OR function))]; JSTOR [(pollination OR pollinator) AND (agroecology OR agroecosystems) AND (effect OR function)], y PubMed [(pollination OR pollinator) AND (agroecology OR agroecosystems) AND (effect OR function)].

Las citas encontradas, junto con su respectivo resumen, fueron importadas al software de gestión de referencias Mendeley, el cual permitió realizar la comprobación de resultados duplicados entre las distintas bases de datos y su posterior eliminación. El protocolo de búsqueda fue aplicado por tres revisores independientes.

\section{Criterios de inclusión y exclusión}

Se incluyeron únicamente artículos originales escritos en inglés, publicados en los últimos diez años (2005-2015), en los cuales el objetivo principal del estudio fuese la valoración del efecto y/o de la función de los polinizadores y/o de la polinización en sistemas de producción agrícola, y que, además, mencionasen algún género, especie o grupo de polinizadores concreto.

Se excluyeron aquellos artículos en los que el efecto y/o la función de la polinización fuese llevado a cabo por especies introducidas por el investigador en el sistema productivo, y no por especies presentes previamente en dicho sistema.

Para poder evaluar la elegibilidad de cada publicación, así como realizar un posterior análisis de los datos extraídos de cada una de ellas, éstos fueron incorporados a una base de datos propia, en la que se anotó la información referente a: título de la publicación, país dónde se desarrolló el estudio, revista, base de datos, criterios de inclusión y criterios de exclusión.

Con el fin de aumentar la exhaustividad de la investigación, también se realizó una búsqueda de literatura gris en Google Académico, aplicando la ruta general de búsqueda y teniendo en cuenta los criterios de inclusión y exclusión. Por último, se llevó a cabo un análisis de todas y cada una de las publicaciones obtenidas tras el proceso completo de búsqueda.

\section{Resultados y Discusión}

La aplicación del protocolo de búsqueda a través de las rutas descritas previamente, cuyas diferentes etapas pueden observarse en la Figura 1, dió como resultado un total de 342 artículos publicados en el rango de años comprendido entre 2005 y 2015. Tras la incorporación de las referencias de los artículos a Mendeley (software de gestión de referencias), la eliminación de duplicados y la aplicación de criterios de inclusión y exclusión, quedaron un total de 14 publicaciones que fueron incluidas en la revisión sistemática. Posteriormente, a esos 14 artículos se les sumaron por exhaustividad otras 6 publicaciones originales no indexadas en las bases de datos, procedentes del buscador genérico Google Académico, las cuales se ajustaban también a los criterios de inclusión y exclusión.

\section{Distribución de las publicaciones según el país de origen, año de publicación y base de datos}

La búsqueda sistemática de la literatura científica publicada entre los años 2005 a 2015, resultó en un total de 20 artículos científicos basados en estudios realizados en 10 países distintos (Figura 2), sobre los cuales destacan Estados Unidos y Brasil, por contar con un mayor número de estudios realizados en sus territorios, con un $35 \%$ y un $17 \%$ respectivamente, seguidos de México (13\%) e Indonesia (8\%). También en la Figura 2, puede observarse el tipo de cultivo mayoritario en cada uno de los países objeto de estudio (Encyclopedia of the Nations, 2015a-k), siendo un 60\% del total de estos dependiente de la polinización animal para su correcto desarrollo, mientras que el $40 \%$ restante requiere de la polinización por el viento o de la autopolinización (McGregor, 1976). 


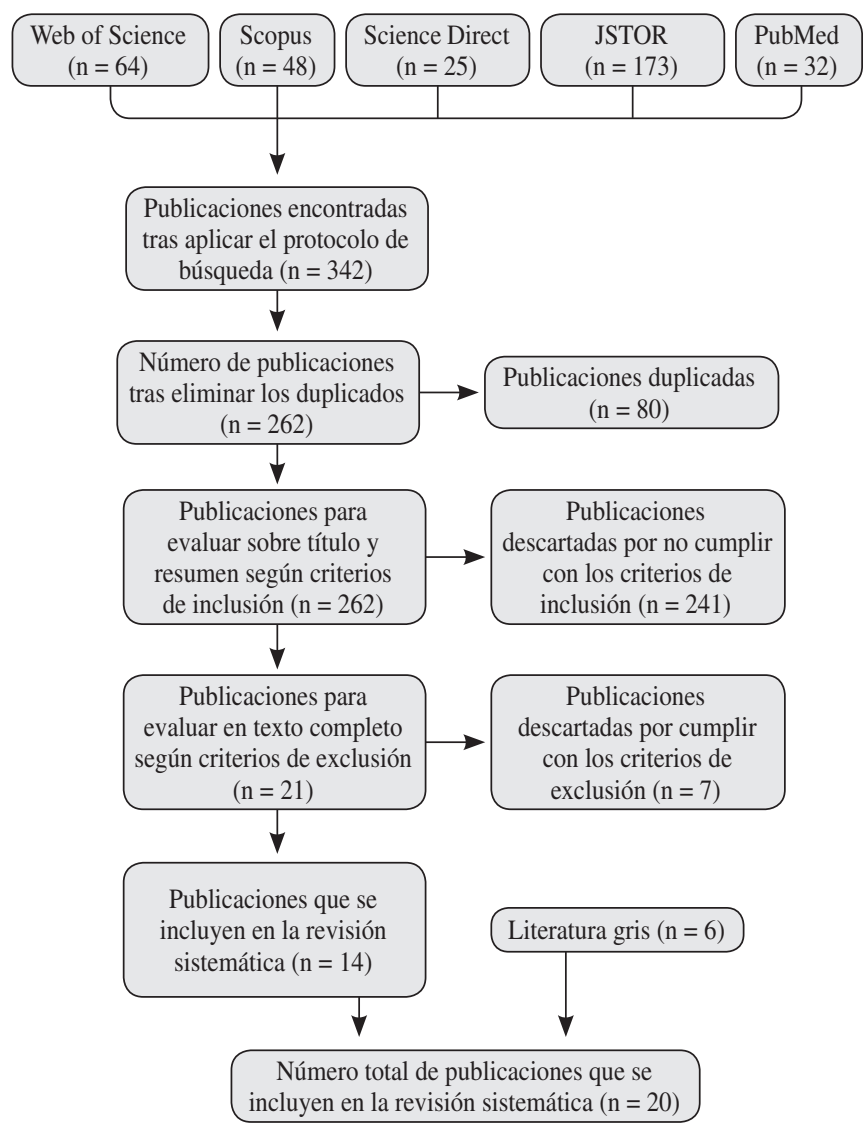

Figura 1. Esquema del protocolo de búsqueda.

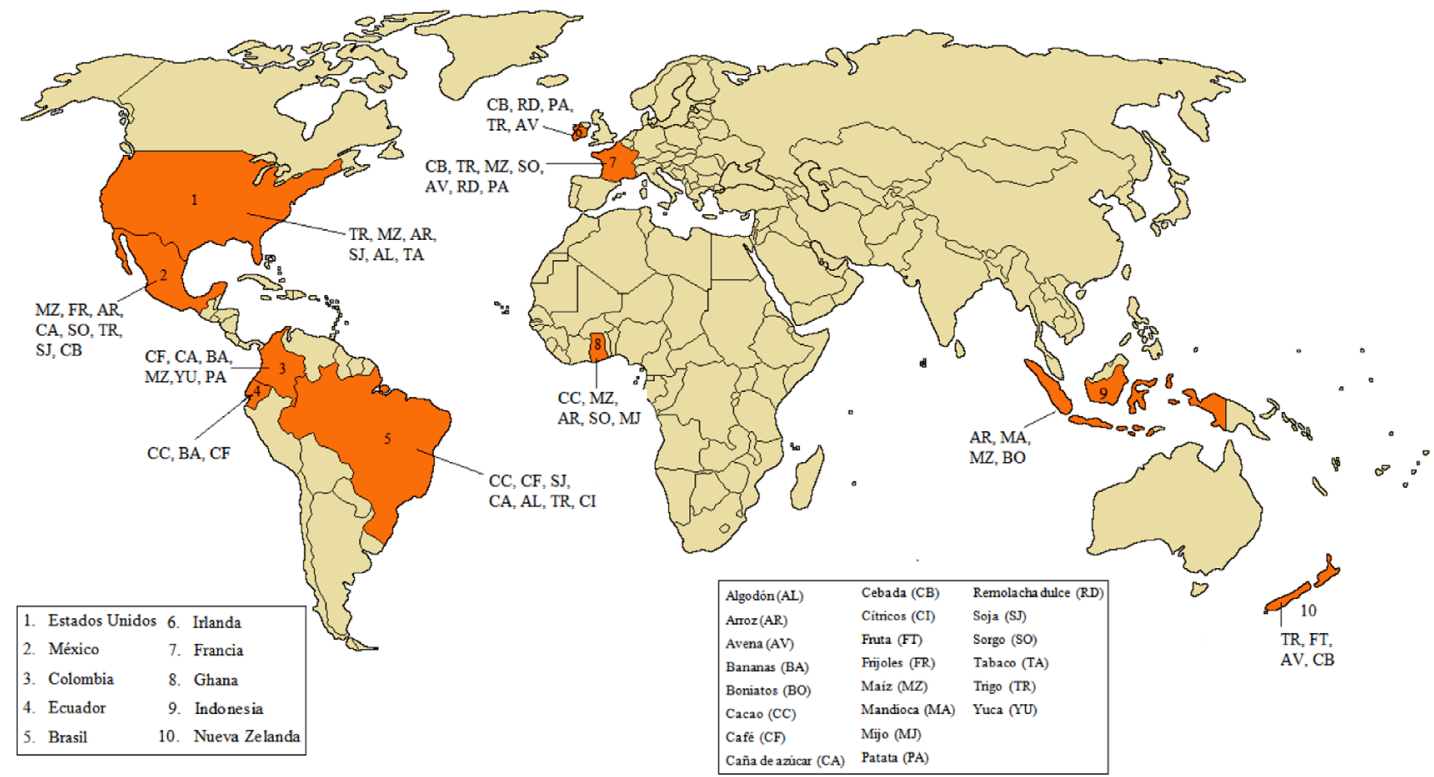

Figura 2. Países de origen de las publicaciones encontradas en la revisión sistemática y tipos de cultivos predominantes (Encyclopedia of the Nations, 2015a-k). 
En la Figura 3 puede observarse que la mayoría de las investigaciones fueron publicadas durante los años 2011 (15\%), 2013 (20\%) y 2014 (15\%), existiendo una ausencia total de publicaciones durante el año 2010, así como que las publicaciones se localizaron tanto en bases de datos multidisciplinares como especializadas. La temática general de la práctica totalidad de las revistas de las cuales proceden los estudios analizados se localiza dentro del área de conocimiento de las ciencias biológicas, agrícolas y ecológicas.

\section{Objetivos, metodologías de investigación y características de la zona de estudio}

A pesar de que todas las publicaciones analizadas en esta revisión parten de la misma base investigativa, determinada por la pregunta de investigación y por el cumplimiento de los criterios de inclusión y exclusión, el objetivo de los distintos estudios varía, pudiéndose organizar, a grandes rasgos, en varios grupos (Tabla 1).

En cuanto a la metodología, al igual que ocurre con los objetivos, las publicaciones pueden agruparse según las técnicas utilizadas para lograr el propósito final de cada estudio. En primer lugar, cabe destacar que la mayoría de las publicaciones se corresponden con estudios experimentales o manipulativos, esto es, aquellos en los que se modifica intencionalmente la zona de estudio, mientras que sólo unos pocos se corresponden con estudios descriptivos, en los
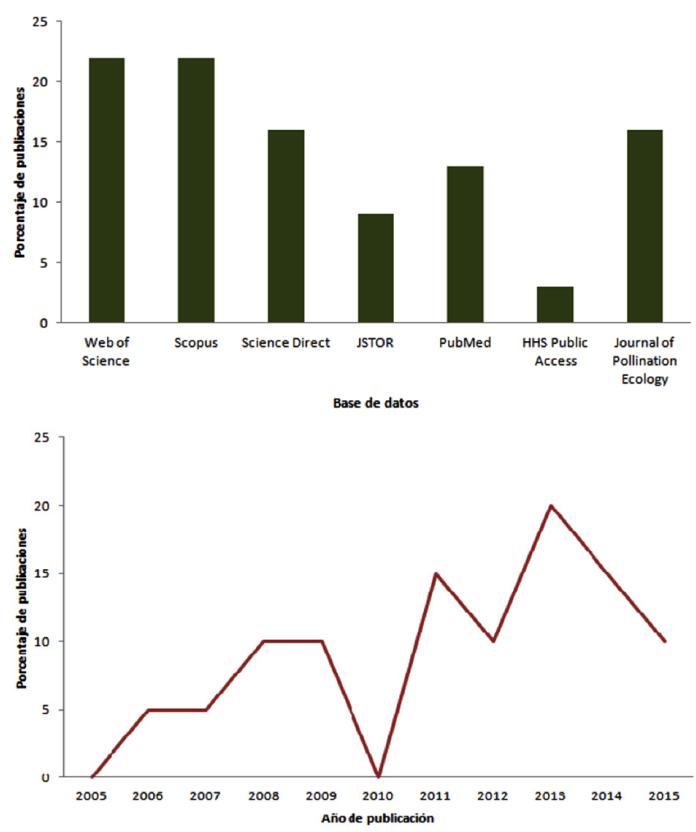

Figura 3. Porcentaje de publicaciones por año y por base de datos.

que la información se obtiene sin modificar el entorno (Tabla 2). Por otro lado, ya respecto a la metodología en sí misma, nos encontramos con varias situaciones, que también pueden verse en la Tabla 2. De igual forma, todas las investigaciones comparten algunas técnicas comunes de análisis estadístico, observables en la Tabla 3.

Otra característica usual en muchos de los estudios es la época del año durante la cual fueron

Tabla 1. Objetivo de las publicaciones

Objetivo de la investigación

Inventariado de polinizadores o cálculo de diversidad, riqueza y/o abundancia de éstos en una determinada zona, como forma de medir la efectividad de la polinización.

Estudios comparativos entre abejas silvestres y abejas melíferas.

Estudios sobre abejas melíferas.

Evaluación del potencial de especies concretas de polinizadores.

Valorar la polinización a través de la calidad y/o la cantidad de frutos y/o semillas.

Valorar la polinización a través del polen y/o del néctar Evaluación de la polinización a través de un indicador matemático. Parte del estudio basado en la evaluación de los efectos de los distintos cultivos, sistemas de manejo o condiciones ambientales sobre la presencia y diversidad de polinizadores.
Publicaciones

Colteaux et al., 2013; Rogers et al., 2013; Russo et al., 2015; Vergara y Badano, 2009; Vilhena et al., 2012; Wojcik et al., 2008.

Chautá-Mellizo et al., 2012; Mallinger y Gratton, 2015; Mensah y Kudom, 2011; Pérez-Balam et al., 2012; Pires et al., 2014. Badano y Vergara, 2011.

Eliyahu, 2015; Greenleaf y Kremen, 2006; Nienhuis y Stout, 2009; Rader et al., 2013.

Bos et al., 2007; Hoehn et al., 2008.

De Melo e Silva Neto et al., 2013.

Ricou et al., 2014.

Badano y Vergara, 2011; Greenleaf y Kremen, 2006; Hoehn et al., 2008; Mallinger y Gratton, 2015; Pires et al., 2014; Rader et al., 2013; Ricou et al., 2014; Vergara y Badano, 2009; Vilhena et al., 2012; Wojcik et al., 2008. 
Tabla 2. Metodología de investigación

Metodología de la investigación

Estudios experimentales o manipulativos.

Estudios descriptivos.

Recolección e identificación de ejemplares de polinizadores presentes en la zona de estudio y/o del número de visitas que realizan a las flores, para obtener datos acerca de la riqueza, abundancia o grupos funcionales mayoritarios.

Recolección de polen o néctar para evaluar la polinización a través de su calidad y/o cantidad.

Recolección de frutos y/o semillas para medir la eficacia de los polinizadores sobre la producción final del cultivo.

Toma de medidas corporales de los polinizadores para evaluar su potencial como tales.

Comparación de medidas de distintos tipo de polinizción (autopolinización, manual polinización cruzada, polinización abierta y exclusión de polinización por embolsado de flores).

Medida de la influencia de los ecosistemas circundantes y de la disponibilidad de recursos alimenticios y de nidificación sobre las comunidades de polinizadores. Medida del impacto de la presencia de abejas melíferas sobre las comunidades de polinizadores nativos o el desarrollo de los cultivos.
Publicaciones

Badano y Vergara, 2011; Bos et al., 2007; Chautá-Mellizo et al., 2012; De Melo e Silva Neto et al., 2013; Eliyahu, 2015; Greenleaf y Kremen, 2006; Hoehn et al., 2008; Mallinger y Gratton, 2015; Mensah y Kudom, 2011; Nienhuis y Stout, 2009; Pérez-Balam et al., 2012; Pires et al., 2014; Rader et al., 2013; Rogers et al., 2013; Vergara y Badano, 2009. Colteaux et al., 2013; Ricou et al., 2014; Russo et al., 2015; Vilhena et al., 2012; Wojcik et al., 2008.

Badano y Vergara, 2011; Colteaux et al., 2013; De Melo e Silva Neto et al., 2013; Eliyahu, 2015; Greenleaf y Kremen, 2006; Hoehn et al., 2008; Mallinger y Gratton, 2015; Mensah y Kudom, 2011; Nienhuis y Stout, 2009; Pérez-Balam et al., 2012; Pires et al., 2014; Rader et al., 2013; Ricou et al., 2014; Rogers et al., 2013; Russo et al., 2015; Vergara y Badano, 2009; Vilhena et al., 2012; Wojcik et al., 2008.

De Melo e Silva Neto et al., 2013; Eliyahu, 2015; Greenleaf y Kremen, 2006; Mensah y Kudom, 2011; Nienhuis y Stout, 2009; Pérez-Balam et al., 2012; Rader et al., 2013; Vilhena et al., 2012.

Badano y Vergara, 2011; Chautá-Mellizo et al., 2012; De Melo e Silva Neto et al., 2013; Greenleaf y Kremen, 2006; Hoehn et al., 2008; Mallinger y Gratton, 2015; Mensah y Kudom, 2011; Nienhuis y Stout, 2009; Pires et al., 2014; Rogers et al., 2013; Vergara y Badano, 2009.

Hoehn et al., 2008; Nienhuis y Stout, 2009.

Bos et al., 2007; Chautá-Mellizo et al., 2012; De Melo e Silva Neto et al., 2013; Eliyahu, 2015; Greenleaf y Kremen, 2006; Hoehn et al., 2008; Mallinger y Gratton, 2015; Mensah y Kudom, 2011; Nienhuis y Stout, 2009; Pérez-Balam et al., 2012; Pires et al., 2014; Rogers et al., 2013; Vergara y Badano, 2009

Badano y Vergara, 2011; Greenleaf y Kremen, 2006; Hoehn et al., 2008; Mallinger y Gratton, 2015; Pires et al., 2014; Rader et al., 2013; Ricou et al., 2014; Vergara y Badano, 2009; Vilhena et al., 2012; Wojcik et al., 2008. Badano y Vergara, 2011; Chautá-Mellizo et al., 2012; Mallinger y Gratton, 2015.

Fuente: Autoría propia.

Tabla 3. Técnicas de análisis estadístico

\begin{tabular}{|c|c|}
\hline Características zona de estudio & Publicaciones \\
\hline $\begin{array}{l}\text { Praderas con distintas especies vegetales, funda- } \\
\text { mentalmente herbáceas o arbustos silvestres }\end{array}$ & $\begin{array}{l}\text { Colteaux et al., 2013; Eliyahu, 2015; Hoehn et al., 2008; Mensah y Kudom, } \\
\text { 2011; Nienhuis y Stout, 2009; Ricou et al., 2014; Wojcik et al., } 2008\end{array}$ \\
\hline $\begin{array}{l}\text { Zonas cultivadas con bordes naturales o } \\
\text { transformados }\end{array}$ & $\begin{array}{l}\text { Badano y Vergara, 2011; Bos et al., 2007; Greenleaf y Kremen, 2006; Hoehn } \\
\text { et al., 2008; Mallinger y Gratton, 2015; Pires et al., 2014; Vilhena } \text { et al., 2012; } \\
\text { Pérez-Balam } \text { et al., } 2012\end{array}$ \\
\hline Huertos tradicionales u orgánicos & $\begin{array}{l}\text { Badano y Vergara, 2011; Bos et al., 2007; De Melo e Silva Neto et al., 2013; } \\
\text { Greenleaf y Kremen, 2006; Hoehn et al., 2008; Mallinger y Gratton, 2015; } \\
\text { Pérez - Balam et al., 2012; Pires et al., 2014; Russo et al., 2015; Vergara y } \\
\text { Badano, 2009; Vilhena } \text { et al., } 2012\end{array}$ \\
\hline Cultivos comerciales o intensivos & $\begin{array}{l}\text { Badano y Vergara, 2011; Chautá-Mellizo et al., 2012; Hoehn } \text { et al., 2008; Pires } \\
\text { et al., 2014; Rader } \text { et al., 2013; Rogers et al., 2013; Vergara y Badano, } 2009\end{array}$ \\
\hline $\begin{array}{l}\text { Zonas cultivadas sometidas a distintas intensidades } \\
\text { de manejo agrícola }\end{array}$ & $\begin{array}{l}\text { Badano y Vergara, 2011; Bos et al., 2007; Chautá-Mellizo et al., 2012; Hoehn } \\
\text { et al., 2008; Pires et al., 2014; Vergara y Badano, } 2009\end{array}$ \\
\hline
\end{tabular}

Fuente: Autoría propia. 
realizados, mostrando una predominancia hacia la estación más cálida del año o la época de floración del cultivo. La duración media de las investigaciones en campo también es común a casi todas ellas, siendo ésta de uno o dos años. Por último, respecto a las características de la zona de estudio, encontramos diversos tipos de ecosistemas, descritos en la Tabla 4.

\section{Grupos de polinizadores, relaciones entre ellos, y rasgos físicos y comportamentales que influyen en su potencial como tales}

En la mayoría de las publicaciones revisadas, al hablar de polinizadores principalmente se hace referencia a aquellos insectos que se encargan de polinizar los cultivos y, más concretamente, la atención suele centrarse en dos grupos, las abejas de la miel (Apis mellifera) y los polinizadores silvestres (abejas y abejorros), dando importancia en ocasiones también a los dípteros. Si bien, existen otros grupos que pueden llevar a cabo esta función, como puede observarse en la Figura 4. Dentro del grupo de los polinizadores silvestres, los géneros con más importancia son Bombus y Xylocopa para abejorros, y Megachile, Lasioglossum, Halictus, Andrena, Ceratina y Trigona para abejas.

Dentro de un agroecosistema, podemos encontrar una variada comunidad de insectos polinizadores, los cuales varían en morfología (distribución y estructura del pelo, longitud de la probóscide), ciclo de vida (incluyendo tamaño de la colonia y fenología) y comportamiento forrajeador (patrones de visitación, constancia floral y estrategias de manejo floral) (Rogers et al., 2013), siendo estas diferencias una parte influyente en la estabilidad y resiliencia de los servicios de polinización de los cultivos (Rader et al., 2013; Rogers et al., 2013). A mayor rango de características comportamentales y roles funcionales, la capacidad de resiliencia de un cultivo se verá incrementada por una mayor capacidad de respuesta a los cambios o alteraciones en el entorno

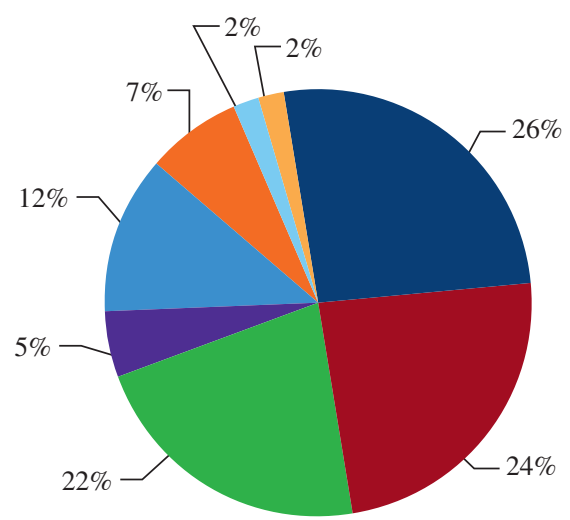

Apis mellifera Abejas silvestres $\square$ Abejorros

Avispas Dípteros Coleópteros Lepidópteros Tisanópteros

Figura 4. Grupos de polinizadores más estudiados.

Tabla 4. Características de la zona de estudio

Técnica estadística utilizada

Transformación logarítmica de los datos.

Análisis de regresión lineal simple.

Análisis de regresión lineal múltiple.

Correlación de Pearson.

Prueba t de Student.

Anova unifactorial.

Anova dos factores.

Modelos lineales generalizados (GLMs).

Modelos de efectos mixtos lineales. Uso del software R.
Publicaciones

Chautá-Mellizo et al., 2012; Eliyahu, 2015; Pérez-Balam et al., 2012; Rader et al., 2013; Ricou et al., 2014; Vilhena et al., 2012.

Badano y Vergara, 2011; De Melo e Silva Neto et al., 2013; Mallinger y Gratton, 2015; Pires et al., 2014; Ricou et al., 2014.

Greenleaf y Kremen, 2006; Hoehn et al., 2008; Nienhuis y Stout, 2009; Vergara y Badano, 2009.

Eliyahu, 2015; Nienhuis y Stout, 2009; Ricou et al., 2014; Vilhena et al., 2012.

De Melo e Silva Neto et al., 2013; Pires et al., 2014; Rader et al., 2013; Vergara y Badano, 2009.

Badano y Vergara, 2011; Chautá-Mellizo et al., 2012; Nienhuis y Stout, 2009; PérezBalam et al., 2012; Vergara y Badano, 2009; Vilhena et al., 2012.

Chautá-Mellizo et al., 2012; Pérez-Balam et al., 2012; Vergara y Badano, 2009; Vilhena et al., 2012.

Bos et al., 2007; Chautá-Mellizo et al., 2012; Hoehn et al., 2008; Mallinger y Gratton, 2015; Nienhuis y Stout, 2009; Rogers et al., 2013.

Eliyahu, 2015; Mallinger y Gratton, 2015; Russo et al., 2015.

Badano y Vergara, 2011; Eliyahu, 2015; Hoehn et al., 2008; Pires et al., 2014; Rader et al., 2013; Ricou et al., 2014. 
(Rader et al., 2013). Sin embargo, también puede ocurrir que, debido a la gran diversidad de respuestas, aparezcan taxones funcionalmente redundantes y se produzca una complementariedad taxonómica, que puede tener lugar tanto a escala espacial como temporal (Hoehn et al., 2008; Mallinger y Gratton, 2015), y tanto a escala de comunidad (p.e repartición de nichos como resultado de diferentes condiciones microclimáticas), como a escala de planta o de polinizador (p.e. diferentes polinizadores visitan diferentes plantas o partes de flores), y que supone que los servicios de polinización obtenidos sean mayores que aquellos provistos por cada taxón de forma aislada (Hoehn et al., 2008; Rader et al., 2013; Rogers et al., 2013).

Algunos estudios tratan sobre la repartición de nicho entre los polinizadores, centrándose en la relación entre las complejas formas de las flores y las distintas especializaciones corporales de los insectos para poder utilizar los recursos florales (Hoehn et al., 2008; Nienhuis y Stout, 2009; Ricou et al., 2014). Por ejemplo, los abejorros suelen forrajear preferentemente en flores cuya corola es tubular, frente a otras planas o convexas, puesto que la longitud de su probóscide les permite tener un mejor acceso al interior de estas profundas estructuras (Hoehn et al., 2008; Ricou et al., 2014). En cambio, aquellas especies de abejas, tales como las abejas melíferas u otras especies silvestres cuya lengua es corta, se ven más atraídas por flores con corolas no tubulares (Greenleaf y Kremen, 2006; Mallinger y Gratton, 2015; Rogers et al., 2013). También en el caso de las abejas de la miel, su incapacidad para sonicar o vibrar para extraer el polen, limita su acceso a determinadas especies vegetales, haciendo de ellas unos polinizadores pobres para algunos cultivos (Greenleaf y Kremen, 2006; Rogers et al., 2013), mientras que, en el caso de los abejorros, su perfecta capacitación para realizar una polinización sonicada, junto con los abundantes y ramificados pelos que recubren sus cuerpos, hacen de ellos unos polinizadores altamente eficientes, que ponen fácilmente en contacto el polen recolectado con los estigmas (Mallinger y Gratton, 2015; Pires et al., 2014; Rader et al., 2013). Otro ejemplo es el de las abejas de la tribu Centridini, que obtienen recursos, principalmente aceite, de las flores de Acerola (Malpighia emarginata). Para ello, las hembras sujetan la parte posterior del pétalo de la flor con sus mandíbulas y raspan los elaióforos, o glándulas secretoras de aceite, moviendo alternativamente las patas delanteras y medias, siendo el aceite así recolectado transportado en una estructura de las patas traseras denominada escopa. Durante el proceso de recolección, la parte ventral del cuerpo de las abejas entra en contacto con las anteras y los estigmas de las flores, constituyéndolas como unos efectivos polinizadores (Vilhena et al., 2012).

También hay estudios que centran su investigación en los distintos patrones de actividad de los polinizadores durante sus visitas a las flores para obtener recursos, considerando que dichos patrones se relacionan con el tamaño corporal del insecto y su habilidad termorreguladora, y que las variaciones en estas características pueden afectar al tiempo y a la duración de las actividades diarias, de manera que diferentes especies responden de forma distinta a las mismas condiciones ambientales (Mallinger y Gratton, 2015; Rader et al., 2013).

Es el caso de moscas y abejas. Existen marcadas diferencias entre la morfología y el comportamiento de estos dos taxones de insectos, que resulta en patrones espacio-temporales de actividad diferentes (Rader et al., 2013). Las abejas tienen mayores requerimientos energéticos, como resultado de realizar un vuelo endotérmico, de la construcción de nidos y del aprovisionamiento de la puesta (Rader et al., 2013), que se traducen en una distribución temporal del forrajeo en ciertos tiempos de actividad diaria, así como a ciertas alturas (Mallinger y Gratton, 2015), probablemente para reducir el gasto energético implicado en el proceso (Hoehn et al., 2008). Además, muchos estudios sostienen que no sólo las características morfológicas, tales como el tamaño corporal, son responsables de la actividad forrajeadora de las abejas, sino que también el tiempo que tarda el polen en liberarse influye en la actividad circadiana de estos himenópteros (Hoehn et al., 2008). Igualmente, hay excepciones a estos patrones generales, siendo el caso de las abejorros del género Bombus, cuyo tamaño corporal es relativamente grande y presentan el cuerpo cubierto de abundantes, largas y densas setas, lo que les permite retener calor durante el forrajeo en condiciones frías, pudiendo estar activos en un rango más amplio de temperaturas (Mallinger y Gratton, 2015; Rader et al., 2013).

Por el contrario, las moscas tienen requerimientos energéticos menores que las abejas, dado que operan ectotérmicamente, pudiendo estar activas a temperaturas frías, y, aunque muchas de ellas visitan las flores para alimentarse de polen y néctar, las 
necesidades de sus larvas varían significativamente entre taxones. Estas características hacen de las moscas unos polinizadores altamente efectivos, puesto que aumentan la posibilidad de que una flor sea visitada a lo largo de un día. Además, puesto que en algunas zonas y/o intervalos de tiempo, las moscas son más abundantes que las abejas, su papel como polinizadores de cultivos podría estar ganando importancia, pudiendo mejorar la respuesta de las comunidades locales ante cambios ambientales y llegando a ser los polinizadores dominantes en caso de que se extinguiesen las abejas (Rader et al., 2013).

Sin embargo, suponiendo que esta situación tuviese lugar, se aseguraría el proceso de la polinización, pero no su efectividad, debido a las diferencias en la eficiencia de los distintos polinizadores y a su distinta contribución a la polinización de un cultivo (Rader et al., 2013; Rogers et al., 2013). De hecho, se ha demostrado que los servicios aportados por los polinizadores son mucho más eficientes cuando tienen lugar interacciones comportamentales entre taxones, como es el caso de algunos cultivos en los que las interacciones entre abejas melíferas y otros polinizadores silvestres incrementan la polinización, aumentando la frecuencia de visitas de estas abejas entre las flores $\mathrm{y}$, por tanto, mejorando la transferencia de polen (Hoehn et al., 2008). Aun así, la relación entre las abejas melíferas y los polinizadores silvestres no siempre es positiva, tal y como se verá más adelante en esta revisión.

Por último, algunos estudios demuestran que la rotación de especies a lo largo de un día sería beneficiosa para el éxito de la polinización y, no sólo en términos de un incremento en la cantidad de polen transferido, sino también en cambios en su distribución, puesto que las abejas grandes que aparecen a horas tempranas transportan más polen, mientras que las pequeñas y tardías mejoran la transferencia de polen entre flores debido a sus movimientos característicos. Además, el cambio temporal de especies polinizadoras también podría mejorar la producción de semillas debido a la combinación de los dos rasgos anteriores (Hoehn et al., 2008). Es por esto que resulta esencial entender las diferencias comportamentales diurnas en la función de los polinizadores, para así poder realizar una evaluación más precisa de los servicios de polinización provistos por las diversas comunidades de cara a futuros cambios ambientales (Rader et al., 2013).

\section{Asociaciones polinizador-planta}

La polinización está estrechamente relacionada con la diversidad funcional de polinizadores, basada, a su vez, en las características especieespecíficas que dirigen el uso complementario de los recursos florales (Hoehn et al., 2008). Es decir, los polinizadores responden a un conjunto de estímulos y recompensas que caracterizan a las flores, y su forrajeo está determinado por rasgos tales como la morfología de la flor y su color, por los que se ven atraídos desde grandes distancias, o por su olor, siendo éste el estímulo predominante en distancias cortas (Ricou et al., 2014).

Muchos estudios sugieren que los polinizadores tienen preferencias por el color de las flores sobre las que forrajean, de tal forma que, por ejemplo, las abejas y los abejorros se ven más atraídos por el color azul y el morado, mientras que los sírfidos prefieren el blanco o el amarillo. De hecho, se cree que algunas especies de plantas son más atractivas para los insectos que otras, como las amapolas, debido a que crean patrones de rayos UV sobre su superficie para atraerlos y que, posiblemente, son usados por los insectos como señales para distinguir entre especies de plantas, o por éstas como forma de discriminación entre especies de insectos (Ricou et al., 2014).

Los polinizadores también muestran preferencias por las inflorescencias frente a las flores solitarias y por las flores simétricas frente a las asimétricas, estando la velocidad de manipulación de una flor determinada por la simetría floral (Ricou et al., 2014). Asimismo, también se establecen relaciones entre la morfología de las corolas y la de los insectos polinizadores, optimizándose el forrajeo, por ejemplo, cuando la longitud de la lengua del polinizador es igual a la profundidad de la corola (Ricou et al., 2014) o cuando la morfología corporal del polinizador encaja en proporción con la morfología de la flor forrajeada (Mensah y Kudom, 2011; Nienhuis y Stout, 2009; Pires et al., 2014).

La actividad de forrajeo y la búsqueda de polen y néctar también se relacionan con el tamaño floral, disminuyendo enormemente a medida que el tamaño se incrementa. El polen es un importante recurso para los polinizadores, puesto que les proporciona cerca del 20\% de los carbohidratos que necesitan y el total de proteínas. Por ello, su abundancia influye en las decisiones de forrajeo del polinizador y su déficit puede afectar a su actividad diaria, tal como 
ocurre con las abejas nodriza y sus cuidados para con las larvas. En cuanto al néctar, proporciona el $80 \%$ de los requerimientos de azúcar de los polinizadores, siendo su principal fuente de energía, y cuya calidad y concentración de azúcares influyen en las especies que forrajean sobre las distintas flores, existiendo plantas sacarosa-dominantes, glucosa-dominantes o fructosa-dominantes, y otras con un contenido balanceado de los tres azúcares. Un ejemplo de las preferencias florales según el néctar sería el caso de las abejas de la miel, que suelen forrajear sobre plantas con un contenido balanceado en azúcares, mientras que los abejorros prefieren una mezcla de los tres azúcares (Ricou et al., 2014).

Respecto al olor floral, este rasgo constituye un importante método de atracción y normalmente consiste en una compleja mezcla de compuestos químicos volátiles pertenecientes a diferentes clases químicas. En el caso de las abejas, el sentido del olfato se encuentra altamente desarrollado y se asocia con altos valores de forrajeo sobre flores con un olor particular o con un conjunto de olores (Ricou et al., 2014).

Por otro lado, en relación con los efectos que desencadenan los polinizadores sobre las plantas, múltiples estudios demuestran que la producción de semillas y frutos se ve incrementada con la presencia de estos organismos, debido a la existencia de una correlación positiva entre su diversidad y la deposición de polen, así como por una serie de factores tales como la dinámica poblacional de las especies, su eficiencia polinizadora, la competitividad intra e interespecífica, la distancia entre parches florales, la disponibilidad de recursos o los sistemas de manejo utilizados (Chautá-Mellizo et al., 2012; Colteaux et al., 2013; De Melo e Silva Neto et al., 2013; Greenleaf y Kremen, 2006; Hoehn et al., 2008; Mallinger y Gratton, 2015; Mensah y Kudom, 2011; Russo et al., 2015; Vergara y Badano, 2009).

La calidad y cantidad de polen, así como la forma en la que es depositado, puede afectar a la producción de frutos y semillas y a las características de la descendencia. Por ejemplo, la deposición de polen procedente de la propia planta o de otras relacionadas puede resultar en una depresión endogámica de rasgos de desarrollo, incluyendo rango de germinación, crecimiento y supervivencia, en comparación con una deposición de polen cruzada. Así mismo, la inadecuada deposición de polen procedente de una polinización cruzada también podría tener efectos negativos inmediatos sobre la producción de frutos y semillas, lo que ha dado lugar a la generación de distintos mecanismos de asociación polinizador-planta para evitar estas situaciones (Chautá-Mellizo et al., 2012)

Algunas especies de plantas con flor, como el aguacate, el algodón o el café, son autógamas y no dependen de la actividad polinizadora para su desarrollo, aunque también se ven beneficiadas por los servicios que esta provee, viendo aumentada su producción (Badano y Vergara, 2011; Bos et al., 2007; Greenleaf y Kremen, 2006; Pérez-Balam et al., 2012; Pires et al., 2014; Vergara y Badano, 2009). En cambio otras, como las calabazas o las manzanas, presentan autoincompatibilidad del polen, requiriendo de la polinización cruzada y siendo estrictamente dependientes de los polinizadores (Greenleaf y Kremen, 2006; Mensah y Kudom, 2011). En otros casos, las especies vegetales requieren de la polinización para su desarrollo, pero, debido a las características morfológicas de sus flores, ésta no puede ser polinizadas por cualquier insecto, como ocurre en el caso de algunas plantas aromáticas que requieren ser polinizadas por insectos de muy pequeño tamaño, como los trips (Eliyahu et al., 2015), o de cultivos como el tomate, cuyo polen solamente es accesible para aquellas abejas capaces de sonicar (De Melo e Silva Neto et al., 2013; Greenleaf y Kremen, 2006).

Por último, ciertos estudios que evalúan la polinización a través de experimentos de manipulación de la misma, muestran en prácticamente todos los casos que el incremento de la producción es siempre mayor tras una polinización natural cruzada, seguido de la polinización manual, y que la ausencia de polinización supone la ausencia de producción (Bos et al., 2007; Chautá-Mellizo et al., 2012; Mensah y Kudom, 2011). De esta forma, los beneficios del incremento manual de la polinización pueden ser compensados en casos en que los recursos limiten la habilidad de la planta para desarrollar semillas o el aumento de la producción de semillas se produzca a expensas de la calidad individual de cada una de ellas (Chautá-Mellizo et al., 2012), mientras que la polinización natural cruzada aumenta la posibilidad de que cualquier flor reciba polen fértil y se obtenga una elevada producción final (Greenleaf y Kremen, 2006). 


\section{Medidas de la efectividad de la polinización}

La necesidad de cuantificar los servicios aportados por los polinizadores se ha vuelto un hecho cada vez más común, en un esfuerzo por entender cómo funcionan los procesos que sustentan nuestro sistema alimenticio (Rogers et al., 2013). Sin embargo, las comunidades de polinizadores presentes en un cultivo varían a lo largo del tiempo y del espacio, y evaluar de forma precisa los servicios que aportan se convierte en un proceso complejo (Rogers et al., 2013), siendo necesario realizar una valoración acertada del rol que realiza cada especie en el proceso, así como de las medidas, metodologías y herramientas a utilizar para obtener esa información (Rogers et al., 2013; Russo et al., 2015).

La polinización puede ser evaluada desde dos perspectivas. Desde el punto de vista inicial de la comunidad de polinizadores y sus interacciones con el cultivo, y desde el punto de vista final de la producción de frutos y semillas (Rogers et al., 2013), teniendo en cuenta que las primeras medidas se relacionan más directa y cuantitativamente con la polinización, mientras que las segundas son menos sensitivas, debido a la influencia de los numerosos factores abióticos que también intervienen en el proceso polinizador y de producción (Hoehn et al., 2008).

Para el primer caso, la contribución de un polinizador a un cultivo es a menudo medida en términos de abundancia de forrajeo (Rader et al., 2013; Rogers et al., 2013), eficiencia por visita (Rogers et al., 2013, patrones de actividad (PérezBalam et al., 2012; Rogers et al., 2013), rango de visitación (Mensah y Kudom, 2011; Rader et al., 2013; Rogers et al., 2013) y/o influencia interespecífica (Rogers et al., 2013). Cada una de las medidas afecta de manera directa a la cantidad y la calidad de los servicios de la polinización, permitiendo la comparación de la importancia relativa entre los distintos polinizadores (Rader et al., 2013).

La eficiencia por visita es una medida potencialmente sencilla y pueden emplearse varios métodos para obtenerla (Rogers et al., 2013). Una de las metodologías más utilizadas consiste en el embolsado de flores. Cuando la flor está cerrada, se cubre con una bolsa que impide el acceso a ella a los insectos y, en el momento en el que se produce la apertura, la bolsa se retira, permitiendo la polinización. Tras la visita de la flor por un polinizador, ésta puede ser recogida para contar los granos de polen depositados en su estigma, puede ser reembolsada y posteriormente se procederá al conteo del número de semillas, o bien se pueden contar los granos de polen transportados por el visitante floral (Bos et al., 2007; Pérez-Balam et al., 2012; Rader et al., 2013; Rogers et al., 2013). Sin embargo, algunos estudios muestran que no siempre se debe usar la efectividad polinizadora como medida del polen recogido, deposición o producción de semillas, puesto que los polinizadores más abundantes no siempre son los más eficientes. Además, los distintos polinizadores podrían tener patrones complementarios de manejo floral y la eficiencia polinizadora podría depender de su orden de llegada a la flor. Por ejemplo, algunos podrían ser más efectivos transfiriendo polen entre flores mientras que otros podrían distribuir el polen previamente transferido sobre la superficie estigmática (Rogers et al., 2013).

Junto con la eficiencia por visita, la abundancia de polinizadores también es una medida altamente informativa y relativamente simple de obtener, con valores que permiten realizar comparaciones entre especies y establecer un rango de eficacia de polinización. No obstante, ambas medidas por sí solas a menudo son insuficientes para describir el conjunto de formas en las que una comunidad de polinizadores podría contribuir al proceso de la polinización, corriendo el riesgo de que, al centrarse solo en estos dos criterios, se sobre o subestimen ciertas especies y no se reconozca su importancia (Rogers et al., 2013).

Otro de los factores que determinan la efectividad de la polinización es el rango de visitación, cuyas medidas pueden identificar la relación entre polinizadores, plantas y la subsecuente producción de frutos (Mensah y Kudom, 2011). En relación con este, lo habitual es realizar una evaluación del llamado "fruit set" o producción de frutos. Algunos estudios realizan comparaciones entre el "fruit set" inicial, o proporción de flores que han sido exitosamente polinizadas, y el "fruit set" final, o proporción de flores que eventualmente resultan en frutos maduros cosechados. A través de la comparación entre estas dos medidas, se obtienen datos acerca del aborto frutal, o número de frutos perdidos durante el proceso (Bos et al., 2007).

Mas allá de todas esta medidas, Ricou et al., 2014 también considera necesario el uso de indicadores para evaluar el nivel de biodiversidad en tierras cultivadas y monitorear su evolución y los efectos 
de las políticas de manejo agrícola sobre ella. Por ello, y ante la ausencia de indicadores predictivos que ayuden a valorar la situación, diseñaron y desarrollaron uno nuevo para evaluar la polinización, tanto a nivel de especies de flores como a nivel de comunidades de plantas, empleando el valor de la riqueza de especies, la abundancia floral y el período de forrajeo.

\section{Situaciones que afectan a los polinizadores}

Los polinizadores, tanto silvestres como manejados, se ven muy influidos por los cambios en el entorno que les rodea (Colteaux et al., 2013; Greenleaf y Kremen, 2006; Wojcik et al., 2008), debido a su gran dependencia por los recursos florales como sustento y hábitats de nidificación adecuados (Colteaux et al., 2013; Wojcik et al., 2008). Si el hábitat de nidificación de un polinizador sufre alguna modificación, éste se ve incapacitado para continuar viviendo en ese lugar, aunque en la zona haya recursos alimenticios en abundancia (Colteaux et al., 2013; Wojcik et al., 2008), siendo esta una de las causas más importantes en el declive de las poblaciones de polinizadores nativos (Colteaux et al., 2013).

La alteración, modificación o destrucción del hábitat de un polinizador puede deberse a múltiples causas naturales, tales como la escasez de recursos florales (Badano y Vergara, 2011), la presencia estacional de especies vegetales más o menos ricas en polen y néctar (Bos et al., 2007; Nienhuis y Stout, 2009; Ricou et al., 2014), o las condiciones climáticas predominantes (Russo et al., 2015). Sin embargo, en la mayoría de las situaciones, el declive a gran escala de sus poblaciones es consecuencia de cambios antropogénicos en el paisaje (Bos et al., 2007).

Actividades humanas, tales como la intensificación en los usos del suelo, la fragmentación, degradación y pérdida del hábitat, o el uso de agroquímicos, tienen efectos adversos sobre la diversidad de polinizadores (Bos et al., 2007; Greenleaf y Kremen, 2006; Hoehn et al., 2008), habiéndose desarrollado algunos estudios que tratan de valorar estos efectos desde el punto de vista de la respuesta de las abejas a los distintos sistemas de manejo del cultivo, a la pérdida de hábitat natural o al incremento del aislamiento entre parches de hábitat (Badano y Vergara, 2011; Vergara y Badano, 2009), y sus variaciones según el grado de especialización de una especie a los recursos florales, a lo largo del ciclo de vida de un polinizador o entre taxones (Greenleaf y Kremen, 2006). Otros estudios evalúan la influencia de la introducción de especies exóticas como la abeja domesticada Apis mellifera (Badano y Vergara, 2011; Colteaux et al., 2013; Vergara y Badano, 2009).

La introducción de la abeja de la miel o abeja melífera en casi todos los países del mundo, y su domesticación durante más de 400 años, podrían considerarse como uno de los mayores experimentos incontrolados inducidos por el ser humano (Badano y Vergara, 2011), dada la facilidad de manejo y cría de esta especie, y la asunción acerca de su gran potencial como polinizador y de sus múltiples efectos beneficiosos sobre las cosechas (Badano y Vergara, 2011; Mallinger y Gratton, 2015; Rogers et al., 2013; Vergara y Badano, 2009).

Las abejas de la miel son recolectores masivos de polen y néctar que focalizan toda su actividad en pequeños parches de flores, agotando por completo todos los recursos que estas proveen y desplazando competitivamente a otros polinizadores de sus fuentes de alimento. Además, el proceso de extracción de recursos es dependiente de la densidad de individuos, de manera que su agotamiento se ve incrementado con un mayor número de abejas melíferas visitando las flores (Badano y Vergara, 2011).

En Eurasia, donde Apis mellifera es nativa y ha coexistido con otras especies de abejas a lo largo de toda su historia evolutiva, su altamente eficiente comportamiento forrajeador parece no tener apenas efecto sobre la diversidad de visitantes florales. Sin embargo, en los lugares donde se ha convertido en un especie introducida, sus efectos densidad-dependientes podrían ser responsables, al menos en parte, de la reducción de la diversidad de las poblaciones de polinizadores nativos y de su número de visitas a las plantas silvestres (Badano y Vergara, 2011). Un ejemplo de ello, sería el estudio realizado por Colteaux et al., 2013, en el que se muestra que la presencia de un elevado número de colmenas de abejas melíferas supone un descenso en el potencial reproductor del abejorro nativo Bombus occidentalis en la Costa de California, así como que, años después de las desaparición de todas las colmenas, se produjo una recuperación de las comunidades de esta especie de abejorro.

Las abejas melíferas también podrían alterar los rangos de polinización de las plantas de múltiples formas, afectando consiguientemente al rendimiento 
de los cultivos. En algunos casos, estas abejas añaden sus servicios a los provistos por los polinizadores nativos y aumentan la cosecha, como es el caso de las plantaciones de café en Venezuela. En otros, como en México, ocurre lo contrario, siendo la presencia de colmenas causante de una reducción de la diversidad de polinizadores silvestres sin proveer servicios equivalentes (Badano y Vergara, 2011).

Por todo ello, cada vez es mayor el número de estudios que muestran a esta especie como un polinizador pobre para una gran variedad de cultivos que, además, influye negativamente sobre otras comunidades de polinizadores, a través de mecanismos como la competencia por los recursos, actuando como vectores de enfermedades y parásitos, o modificando el hábitat (Badano y Vergara, 2011; Colteaux et al., 2013). Así mismo, también surgen multitud de trabajos que demuestran que los polinizadores silvestres son capaces de aportar los servicios de polinización necesarios para un cultivo sin necesidad de que haya abejas melíferas presentes (Hoehn et al., 2008), sin incurrir en costes económicos (Vergara y Badano, 2009) y de forma mucho más eficiente que la especie domesticada (Badano y Vergara, 2011; Mallinger y Gratton, 2015). Aún así, los apicultores siguen siendo reacios a depender exclusivamente de estos organismos para polinizar sus cultivos, puesto que la abundancia y diversidad de abejas varía a lo largo de las regiones y las temporadas de crecimiento, resultando en servicios de polinización no confiables (Mallinger y Gratton, 2015).

\section{Medidas de conservación de polinizadores}

Asociada a la progresiva pérdida de biodiversidad en los cultivos, existe una creciente concienciación social encaminada a encontrar medidas de conservación y mantenimiento de hábitats y especies encargados de llevar a cabo servicios ecosistémicos de vital importancia para el correcto funcionamiento de los agroecosistemas (Bos et al., 2007). Para garantizar la prestación de los servicios de polinización silvestre, la atención debería centrarse en la protección de grandes áreas naturales asociadas a los cultivos, dada su capacidad para sostener a estos organismos y actuar como hábitat para ellos, así como en la protección de las especies de polinizadores frente a plagas y enfermedades (De Melo e Silva Neto et al., 2013; Vilhena et al., 2012).
Existen diversas técnicas que pueden ser implementadas para asegurar la conservación y protección de los polinizadores frente a cambios en el entorno, algunas tan sencillas como mantener una alta diversidad floral de especies ricas en polen y néctar (Ricou et al., 2014), tanto dentro de los propios cultivos (Mallinger y Gratton, 2015) como a lo largo de sus márgenes (Ricou et al., 2014; Vilhena et al., 2012), que actúan como atrayentes para los polinizadores $\mathrm{y}$, al mismo tiempo, les proporcionan lugares para anidar y recursos alimenticios y para la construcción del nido (Mallinger y Gratton, 2015; Ricou et al., 2014; Vilhena et al., 2012). Otras técnicas más complejas implicarían sistemas de manejo agrícola que favoreciesen a la diversidad de polinizadores y que podrían resultar en un incremento de la productividad y una mejor conservación de la biodiversidad (Vergara y Badano, 2009). Aparte, algunos autores consideran la posibilidad de desarrollar herramientas de conservación y gestión de comunidades de abejas en zonas urbanas (Wojcik et al., 2008).

Por otra parte, también debería profundizarse en el conocimiento sobre la biología de estos organismos y en las interacciones existentes entre el entorno, los polinizadores silvestres y las abejas domesticadas, con el fin de proponer y desarrollar planes y estrategias de gestión y conservación adecuados para cada especie y situación (Greenleaf y Kremen, 2006; Wojcik et al., 2008). Así mismo, debería considerarse como parte del proceso de conservación la educación del agricultor y/o del consumidor acerca del proceso polinizador, puesto que estos podrían ser un punto clave en la reducción de costos para la producción de alimentos en el camino hacia la seguridad alimentaria (Mallinger y Gratton, 2015; Pérez-Balam et al., 2012).

\section{Áreas emergentes de investigación en polinización}

Dentro del área de investigación en polinización, existen ciertos temas sobre los que, de una u otra forma, se hace mención en casi todos los estudios, tales como los impactos negativos causados por los cambios en el entorno o la introducción de especies exóticas (Chautá-Mellizo et al., 2012; Colteaux et al., 2013; Greenleaf y Kremen, 2006; Ricou et al., 2014; Wojcik et al., 2008); la importancia crítica de invertir recursos a largo plazo para evaluar de forma precisa la diversidad de un ecosistema (Russo 
et al., 2015; Vilhena et al., 2012), especialmente para aquellas especies que juegan una función distintiva como parte de una comunidad (Rader et al., 2013; Russo et al., 2015) o la asociación entre polinizadores y plantas, y sus efectos sobre la productividad de un cultivo (Chautá-Mellizo et al., 2012; Vilhena et al., 2012).

Sin embargo, también existe multitud de temas relevantes sobre los que aún no se conoce nada o que apenas han sido investigados, entre los cuales podríamos encontrar nuevas ideas para desarrollar futuros proyectos en este campo de investigación. Algunas ideas podrían basarse en las propuestas de Chautá-Mellizo et al., 2012 de evaluar los efectos de algún grupo de polinizadores sobre un cultivo concreto, manipulando experimentalmente la polinización y examinando las consecuencias de este servicio sobre la descendencia del cultivo, quizás sobre cultivos de tomate (De Melo e Silva Neto et al., 2013; Greenleaf y Kremen, 2006), algodón (Pires et al., 2014), café (Vergara y Badano, 2009) o aguacate (Pérez-Balam et al., 2012); o como propone Ricou et al., 2014, analizar el porqué de la atracción de los polinizadores por las plantas, a través de la relación entre los rasgos florales y la polinización, quizás por medio de los umbrales de atracción de los polinizadores por las inflorescencias o de la capacidad de los polinizadores de discriminar el polen de acuerdo con su calidad. Así mismo, las propuestas también podrían centrar su atención en evaluar otros polinizadores distintos a las abejas, como son los polinizadores silvestres, y su conservación, tal como plantean Colteaux et al., 2013; Mallinger y Gratton, 2015 y Rader et al., 2013.

\section{Conclusiones}

Los resultados de la revisión sistemática mostraron una cierta tendencia a la realización de investigaciones basadas en el inventariado de polinizadores y la obtención de medidas de diversidad, riqueza y/o abundancia, así como una predominancia de trabajos en los que, más allá de su objetivo principal, también trataban de evaluar los efectos de los distintos sistemas de manejo agrícola sobre la presencia y diversidad de estos organismos.

De igual forma, todos los trabajos analizados compartían ciertas características relacionadas con la metodología empleada, abundando los estudios de tipo experimental o manipulativo, y presentando gran variedad de técnicas de recopilación de información, que implicaban desde la recolección de ejemplares de polinizadores, polen, néctar, semillas o frutos, hasta experimentos de manipulación de la polinización y comparación entre tratamientos. Respecto a la época del año, casi todas las investigaciones en campo fueron realizadas durante la época cálida y a lo largo de un tiempo mínimo de un año, fundamentalmente en dos tipos de ecosistemas, las praderas de vegetación natural y las zonas cultivadas, tanto tradicionales como intensivas.

En cuanto al grupo de polinizadores más frecuentemente estudiado, pudo observarse una mayoría de investigaciones basadas en polinizadores manejados, como la abeja melífera, o en polinizadores silvestres, fundamentalmente abejas y abejorros. En prácticamente todos los casos, se analizó el fenómeno de la repartición de nicho entre grupos funcionales, sus patrones de actividad diaria y las distintas relaciones establecidas entre polinizadores y plantas para tener un acceso más eficiente a los recursos. Destaca también la demostración por parte de algunos trabajos de la asociación existente entre algunos grupos de polinizadores y cultivos concretos, así como de los beneficios que aporta la polinización natural sobre ciertas especies vegetales no necesariamente dependientes de este proceso para su desarrollo.

A la hora de realizar la evaluación de los beneficios aportados, se observó un consenso respecto al uso de medidas directas y cuantitativas, consideradas anteriores al proceso polinizador, tales como abundancia de forrajeo, eficiencia por visita o influencia interespecífica, aunque también se emplearon medidas post-polinización, como cantidad y calidad de polen depositado o producción final de frutos y semillas.

Por último, en todas las publicaciones se mostró una preocupación común por las distintas causas antrópicas que están afectando a las comunidades de polinizadores en todo el mundo, y hacia la necesidad de desarrollar e implementar medidas de conservación que garanticen la adecuada prestación de los servicios de polinización en los ecosistemas y que ayuden a la sociedad a entender mejor este proceso.

De todo ello se concluye que, gracias a la realización de esta revisión sistemática, se han podido observar ciertas tendencias dentro de los estudios realizados en el campo de la polinización, así como una creciente preocupación por la función clave de 
los organismos polinizadores en los sistemas de producción agrícola, a pesar de lo cual sigue siendo necesario el desarrollo de nuevas investigaciones, fundamentalmente orientadas a completar los vacios de conocimiento con los que aún contamos hacia algunos de estos organismos y su relación con los ecosistemas.

\section{Agradecimientos}

La finalización de este trabajo fue posible gracias a la ayuda económica aportada por la Asociación Universitaria Iberoamericana de Posgrado (AUIP) y su programa de "Becas de Movilidad entre Instituciones asociadas a la AUIP 2015".

\section{Literatura Citada}

Badano, E.I.; Vergara, C.H.

2011. Potential negative effects of exotic honey bees on the diversity of native pollinators and yield of highland coffee plantations. Agricultural and Forest Entomology, 13: 365-372.

Bonilla, M.A.

2012. La polinización como servicio ecosistémico. En: Iniciativa colombiana de polinizadores (ICPA), Capítulo I: abejas. Universidad Nacional de Colombia, Instituto Humboldt. Bogotá, Colombia. pp. 1-103.

Bos, M.M.; Veddeler, D.; Bogdanski, A.K.; Klein, A.M.; Tscharntke, T.; Steffan-Dewenter, I.; Tylianakis, J.

2007. Caveats to quantifying ecosystem services: fruit abortion blurs benefits from crop pollination. Ecological Applications, 17 (6): 1841-1849.

Chautá-Mellizo, A.; Campbel, S.A.; Bonilla, M.A.; Thaler, J.S.; Poveda, K.

2012. Effects of natural and artificial pollination on fruit and offspring quality. Basic and Applied Ecology, 13: 524-532.

Colteaux, B.C.; McDonald, C.; Kolipinski, M.; Cunningham, J.B.; Ghosh, S.

2013. A survey of pollinator and plant interactions in meadow and grassland habitats of Marin County, California. BIOS, 84 (1): 1-7.

De Melo e Silva Neto, C.; Gomes Lima, F.; Gonçalves Bastos, B.; Lima Bergamini, L.; Araújo Ribeiro Bergamini, B.; Da Silva Elias, M.A.; Villaron Franceschinelli, E.

2013. Native bees pollinate tomato flowers and increase fruit production. Journal of Pollination Ecology, 11 (6): 41-45.

Eliyahu, D.; McCall, A.C.; Lauck, M.; Trakhtenbrot, A.; Bronstein, J.L.

2015. Minute pollinators: The role of thrips (Thysanoptera) as pollinators of pointleaf manzanita, Arctostaphylos pungens (Ericaceae). Journal of Pollination Ecology, 16: 64-71.

Encyclopedia of the Nations.

2015a. Brazil-Agriculture. Disponible en http://www. nationsencyclopedia.com/Americas/Brazil-AGRICULTURE. html Consultado:08/Dic/2015.

Encyclopedia of the Nations.

2015b. Colombia-Agriculture. Disponible en http:// www.nationsencyclopedia.com/Americas/ColombiaAGRICULTURE.html Consultado:08/Dic/2015.

Encyclopedia of the Nations.

2015c. Ecuador-Agriculture. Disponible en http:// www.nationsencyclopedia.com/Americas/EcuadorAGRICULTURE.html Consultado:08/Dic/2015.

Encyclopedia of the Nations.

2015d. France-Agriculture. Disponible en http://www. nationsencyclopedia.com/Europe/France-AGRICULTURE. html Consultado:08/Dic/2015.
Encyclopedia of the Nations.

2015e. Ghana-Agriculture. Disponible en http://www. nationsencyclopedia.com/Africa/Ghana-AGRICULTURE. html Consultado:08/Dic/2015.

Encyclopedia of the Nations.

2015f. Indonesia-Agriculture. Disponible en http://www. nationsencyclopedia.com/Asia-and-Oceania/IndonesiaAGRICULTURE.html Consultado:08/Dic/2015.

Encyclopedia of the Nations.

2015g. Ireland-Agriculture. Disponible en http://www. nationsencyclopedia.com/Europe/Ireland-AGRICULTURE. html Consultado:08/Dic/2015.

Encyclopedia of the Nations.

2015h. Mexico-Agriculture. Disponible en http:// www.nationsencyclopedia.com/Americas/MexicoAGRICULTURE.html Consultado:08/Dic/2015.

Encyclopedia of the Nations.

2015i. New Zealand-Agriculture. Disponible en http://www. nationsencyclopedia.com/Asia-and-Oceania/New-ZealandAGRICULTURE.html Consultado:08/Dic/2015.

Encyclopedia of the Nations.

2015j. South Africa-Agriculture. Disponible en http:// www.nationsencyclopedia.com/Africa/South-AfricaAGRICULTURE.html Consultado:08/Dic/2015.

Encyclopedia of the Nations.

2015k. United States-Agriculture. Disponible en http:// www.nationsencyclopedia.com/Americas/United-StatesAGRICULTURE.html Consultado:08/Dic/2015.

Fründ, J.; Dormann, C.F.; Holzschuh, A.; Tscharntke, T.

2013. Bee diversity effects on pollination depend on functional complementarity and niche shifts. Ecology, 94 (9): 2042-2054.

Greenleaf, S.S.; Kremen, C.

2006. Wild bee species increase tomato production and respond differently to surrounding land use in Northern California. Biological Conservation, 133: 81-87.

Hoehn, P.; Tscharntke, T.; Tylianakis, J.M.; Steffan-Dewenter, I. 2008. Functional group diversity of bee pollinators increases crop yield. Proceedings of The Royal Society B, 275: 2283-2291.

Klatt, B.K.; Holzschuh, A.; Westphal, C.; Clough, Y.; Smit, I.; Pawelzik, E.; Tscharntke, T.

2014. Bee pollination improves crop quality, shelf life and commercial value. Proceedings of The Royal Society B, 281: 20132440.

Klein, A.M.; Vaissière, B.E.; Cane, J.H.; Steffan-Dewenter, I.; Cunningham, S.A.; Kremen, C.; Tscharntke, T.

2007. Importance of pollinators in changing landscapes for world crops. Proceedings of The Royal Society B, 274: 303-313. 
Kremen, C.; Williams, N.M.; Aizen, M.A.; Gemmill-Herren, B.; LeBuhn, G.; Minckley, R.; Packer, L.; Potts, S.G.; Roulston, T.; Steffan-Dewenter, I.; Vázquez, D.P.; Winfree, R.; Adams, L.; Crone, E.E.; Greenleaf, S.S.; Keitt, T.H.; Klein, A.M.; Regetz, J.; Ricketts, T.S.

2007. Pollination and other ecosystem services produced by mobile organisms: a conceptual framework for the effects of land-use change. Ecology Letters, 10: 299-314.

Mallinger, R.E.; Gratton, C.

2015. Species richness of wild bees, but not the use of managed honeybees, increases fruit set of a pollinator-dependent crop. Journal of Applied Ecology, 52 (2): 323-330.

Mayer, C.; Adler, L.; Armbruster, W.S.; Dafni, A.; Eardley, C.; Huang, S.Q.; Kevan, P.G.; Ollerton, J.; Packer, L.; Ssymank, A.; Stout, J.C.; Potts, S.G.

2011. Pollination ecology in the $21^{\text {stcentury: key questions for }}$ future research. Journal of Pollination Ecology, 3 (2): 8-23.

McGregor, S.E.

1976. Insect pollination of cultivated crop plants. Agricultural Research Service, U.S. Dept. of Agriculture, 6a Ed. Washington D.C. Usa. 411 p.

Mensah, B.A.; Kudom, A.A.

2011. Foraging dynamics and pollination efficiency of Apis mellifera and Xylocopa olivacea on Luffa aegyptiaca Mill (Cucurbitaceae) in Southern Ghana. Journal of Pollination Ecology, 4 (5): 34-38.

Nienhuis, C.M.; Stout, J.C.

2009. Effectiveness of native bumblebees as pollinators of the alien invasive plant Impatiens glandulifera (Balsaminaceae) in Ireland. Journal of Pollination Ecology, 1 (1): 1-11.

Pantoja, A.; Smith-Pardo, A.; García, A.; Sáenz, A., Rojas, F. 2014. Principios y avances sobre polinización como servicio ambiental para la agricultura sostenible en países de Latinoamérica y El Caribe. Organización de las Naciones Unidas para la Alimentación y la Agricultura-FAO. Santiago, Chile.

Pérez-Balam, J.; Quezada-Euán, J.J.G.; Alfaro-Bates, R.; Medina,

S.; McKendrick, L.; Soro, A.; Paxton, R.J.

2012. The contribution of honey bees, flies and wasps to avocado (Persea americana) pollination in Southern Mexico. Journal of Pollination Ecology 8 (6): 42-47.
Pires, V.C.; Silveira, F.A.; Sujii, E.R.; Torezani, K.R.S.; Rodrigues, W.A.; Albuquerque, F.A.; Rodrigues, S.M.M.; Salomão, A.N.; Pires, C.S.S.

2014. Importance of bee pollination for cotton production in conventional and organic farms in Brazil. Journal of Pollination Ecology, 13 (16): 151-160.

Rader, R.; Edwards, W.; Westcott, D.A.; Cunningham, S.A.; Howlett, B.G.

2013. Diurnal effectiveness of pollination by bees and flies in agricultural Brassica rapa: Implications forecosystem resilience. Basic and Applied Ecology, 14: 20-27.

Ricou, C.; Schneller, C.; Amiaud, B.; Plantureux, S.; Bockstaller, C. 2014. A vegetation-based indicator to assess the pollination value of field margin flora. Ecological Indicators, 45: 320-331.

Rogers, S.R.; Tarpy, D.R.; Burrack, H.J.

2013. Multiple Criteria for Evaluating Pollinator Performance in Highbush Blueberry (Ericales: Ericaceae) Agroecosystems. Environmental Entomology,42 (6): 1201-1209.

Russo, L.; Park, M.; Gibbs, J.; Danforth, B.

2015. The challenge of accurately documenting bee species richness in agroecosystems: bee diversity in eastern apple orchards. Ecology and Evolution, 5 (17): 3531-3540.

Vergara, C.H.; Badano, E.I.

2009. Pollinator diversity increases fruit production in Mexican coffee plantations: The importance of rustic management systems. Agriculture, Ecosystems and Environment 129: 117-123.

Vilhena, A.M.; Rabelo, L.; Bastos, E.M.; Augusto, S.C.

2012. Acerola pollinators in the savanna of Central Brazil: temporal variations in oil-collecting bee richness and a mutualistic network. Apidologie, 43: 51-62.

Wojcik, V.A.; Frankie, G.W.; Thorp, R.W.; Hernandez, J.L. 2008. Seasonality in Bees and Their Floral Resource Plants at a Constructed Urban Bee Habitat in Berkeley, California. Journal of the Kansas Entomological Society, 81 (1): 15-28. 